“ (C) 2017 IEEE. Personal use of this material is permitted. Permission from IEEE must be obtained for all other uses, in any current or future media, including

reprinting/republishing this material for advertising or promotional purposes, creating new collective works, for resale or redistribution to servers or lists, or reuse of any copyrighted component of this work in other works." 


\title{
Designing A Pulsed Eddy Current Sensing Setup for Cast Iron Thickness Assessment
}

\author{
Nalika Ulapane*, Linh Nguyen*, Jaime Valls Miro*, Alen Alempijevic* and Gamini Dissanayake* \\ ${ }^{*}$ Centre for Autonomous Systems, Faculty of Engineering and Information Technology, University of Technology Sydney, \\ 81 Broadway, Ultimo NSW 2007, Australia. \\ Email: \{nalika.ulapane, vanlinh.nguyen, jaime.vallsmiro, alen.alempijevic, gamini.dissanayake\}@uts.edu.au
}

\begin{abstract}
Pulsed Eddy Current (PEC) sensors possess proven functionality in measuring ferromagnetic material thickness. However, most commercial PEC service providers as well as researchers have investigated and claim functionality of sensors on homogeneous structural steels (steel grade Q235 for example). In this paper, we present design steps for a PEC sensing setup to measure thickness of cast iron, which is unlike steel, is a highly inhomogeneous and nonlinear ferromagnetic material. The setup includes a PEC sensor, sensor excitation and reception circuits, and a unique signal processing method. The signal processing method yields a signal feature which behaves as a function of thickness. The signal feature has a desirable characteristic of being lowly influenced by lift-off. Experimental results show that the setup is usable for Nondestructive Evaluation (NDE) applications such as cast iron water pipe assessment.
\end{abstract}

\section{INTRODUCTION}

Pulsed Eddy Current (PEC) sensing is widely used for Nondestructive Evaluation (NDE) of metallic structures to estimate material thickness remaining under the sensor footprint [1], [2]. Among different PEC sensor architectures, the detector coil based architecture has been found in literature to be usable to detect ferromagnetic material thickness [3], [4], [5], [6]. However, previous research, even recent ones [3], [4], [5], [6] have been focused on standard structural steels grades such as Q235. Therefore in this paper, we present design steps to develop a PEC sensing setup capable of measuring cast iron thickness up to about $25 \mathrm{~mm}$. Even through we focus on cast iron, the design steps are generic and applicable to design a sensor to operate on any desired material.

The motivation for this work was the requirement for NDE of cast iron critical water pipes [7]. It is generally recognized worldwide that about $70 \%$ of the total asset base of urban water utilities consists of buried pipes [8]. Currently existent and aged buried pipes are typically made of gray cast iron, ductile cast iron and mild steel [2]. Out of these, gray cast iron pipes were usually laid about a century ago and some of them are still operational [2]. However, there is a high risk of these aged cast iron pipes failing in due course causing high expenditure to water utilities, as pipe walls get rusted and graphitized due to the typically existent underground environment [2], [7]. This demands for state of the art developments in NDE sensor technologies to facilitate condition assessment of aged ferromagnetic water pipes, especially cast iron.

In this paper, we start by briefly explaining the operating principle of the detector coil based PEC sensor architecture.
We then proceed sequentially describing the design steps of the sensor excitation circuit, the PEC sensor and the signal acquisition circuit. Next we present experimental signals captured from the setup used on gray cast iron and propose a signal processing method to extract a signal feature which behaves as a function of thickness. This feature has the characteristic of having low influence from lift-off, which is highly desired when considering NDE applications such as pipe inspection. By calibrating for a particular cast iron, the setup exhibits its usability for measuring cast iron thickness, and NDE applications at large.

The paper is structured as follows: Section II explains the PEC sensor operating principle in relation to the detector coil based sensor architecture; Section III presents the design steps of the PEC sensing setup; Section IV presents a unique signal processing method which yields a signal feature which behaves as a function of cast iron thickness; Section V presents experimental results captured from the setup when used on gray cast iron; and Section VI concludes the paper by discussing the implications of results and the possibility of using the setup for cast iron pipe assessment.

\section{Detector Coil Based PeC Sensor Operating PRINCIPLE}

Since we deal with ferromagnetic materials the interest of this paper is the detector coil based PEC sensor architecture. This is the architecture literature [3], [6], [4] has identified to have superior sensitivity to ferromagnetic material thickness, therefore it is the architecture typically used to assess ferromagnetic material thickness.

As shown in Fig. 1, the detector coil based PEC sensor is typically composed of two concentrically wound, air cored, conductive circular coils [3], [6], [4]. Concentrically wound rectangular coils too are rarely used [2], [9]. One coil behaves as an exciter coil while the other behaves as a detector coil which captures the signal. The exciter coil is excited with a voltage pulse which can theoretically be modeled as a Heaviside step function. The pulsed excitation causes a rapid change in the surrounding magnetic field. This change induces eddy currents in the test piece which the sensor is assessing. The induced eddy currents will be varying with time and the net effect of this variation and the excitation pulse induces a unique time varying voltage in the detector coil. This voltage is identified as the PEC signal which carries information about 
the test piece. The PEC signal $V(t)$ can be modeled as an infinite summation of exponentials as in Eq. 1 [4] where $b_{i}$ and $c_{i}$ are constants containing information about properties of the sensor and the test piece, and its typical shape is an exponential decay as shown in Fig. 2.

$$
V(t)=\sum_{i=1}^{\infty} b_{i} \exp \left(-c_{i} t\right)
$$

It has been proven in [2] that the PEC signal feature $\beta$ defined as

$$
\beta=\lim _{t \rightarrow \infty}\left|\frac{\mathrm{d} \ln [V(t)]}{\mathrm{d} t}\right|^{-1},
$$

behaves as a function of ferromagnetic material thickness. Therefore, our objective is to extract the $\beta$ feature from the signals produced by our sensor setup when used on cast iron and examine its functional behavior with thickness.

\section{Design Steps of the PEC Sensing Setup}

Fig. 3 shows the basic block diagram of the sensing setup. It includes a computer (PC), a data acquisition card (DAQ), an excitation circuit, a reception circuit and a PEC sensor interacting with a metal test piece (cast iron for our work).

The DAQ we used was an NI USB-6221 Legacy USB DAQ device which drives the excitation circuit as well as samples and acquires the PEC signals. The PC communicates and controls the DAQ while storing the signals. Any PC which can be interfaced with an NI USB-6221 Legacy USB DAQ device would have been appropriate for this work; the PC we used had an Intel(R) Core(TM) i7-3840QM CPU @ 2.80 GHz,

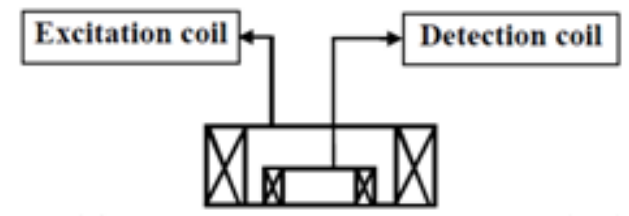

Test Piece

Fig. 1. Cross sectional view of a typical detector coil based PEC sensor.

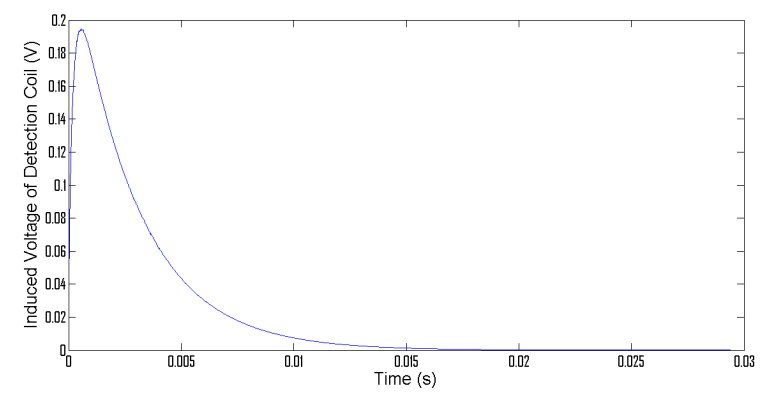

Fig. 2. A typical PEC Signal: Induced voltage in the detection coil.

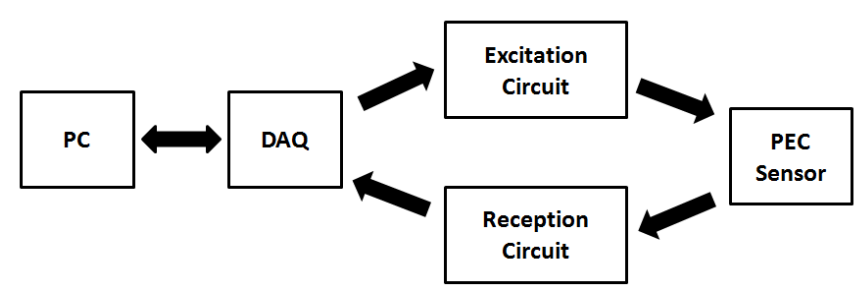

Fig. 3. Block diagram of the sensing setup.

16.0 GB RAM and was running on 64-bit Windows operating system with MATLAB R2013b installed to communicate with the DAQ via "Session Based" interface.

Design details about the excitation circuit, PEC sensor and reception (signal acquisition) are provided from here on in.

\section{A. Excitation Circuit}

From literature it can be seen that a PEC signal is captured for several tens of milliseconds for ferromagnetic material thickness quantification purposes [4]. Therefore we chose to excite the sensor with a pulse having a $100 \mathrm{~ms}$ period and $50 \%$ duty ratio. This enables capturing a signal for a duration of $50 \mathrm{~ms}$. We used an analog output terminal of the DAQ to generate the excitation pulse with an amplitude of $5 \mathrm{~V}$ peak to peak. The PC triggers the DAQ via the MATLAB session based interface to start and stop the excitation signal. Since the DAQ output terminals can supply only low currents, we send the pulse through a power amplification stage. Fig. 4 shows the schematic of the circuit we used for power amplification (a non inverting amplifier with a voltage gain of 2); $V_{s}$ denotes $\pm 15 \mathrm{~V}$ DC supply voltage, $V_{i}$ denotes the $5 \mathrm{~V}$ peak to peak voltage pulse (100 ms period, $50 \%$ duty) generated by the DAQ and $V_{o}$ denotes the amplified pulse. The DAQ generates the pulse from an analog output terminal in reference to the DAQ ground (connected as the $0 \mathrm{~V}$ common ground for the whole setup) denoted by $G N D$ in Fig. 4 . We thus obtained a voltage pulse with an amplitude of $10 \mathrm{~V}$ peak to peak at the amplifier output. We used the OPA544T operational amplifier due to its fast slew rate, high output current supply capability and low output impedance. As we connected the PEC sensor directly to the amplifier output, a low output impedance was desirable since it reduces losses and enables maximum power deliverance to the load which is the PEC sensor in our case.

\section{B. PEC Sensor}

We opted to develop a circular PEC sensor having concentric air cored coils as the exciter and detector as shown in Fig. 1 due to its simplicity and it being widely used in literature for ferromagnetic material assessment [3], [4], [6]. For a fixed excitation, the sensor size has been observed to be a dominant factor influencing the sensor's penetration capability (i.e., the maximum thickness of a particular material the sensor will be sensitive to) [10]. Therefore, before fabrication we simulated the sensor interaction with cast iron using Finite Element Analysis (FEA). We developed a 2D-Axisymmetric model of the sensor placed on cast iron (shown in Fig. 5) 


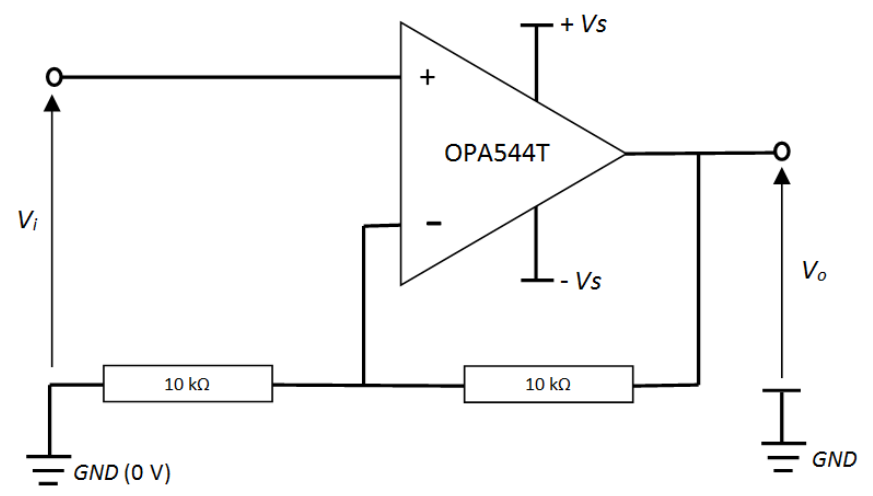

Fig. 4. Schematic of the power amplification stage of the excitation circuit.

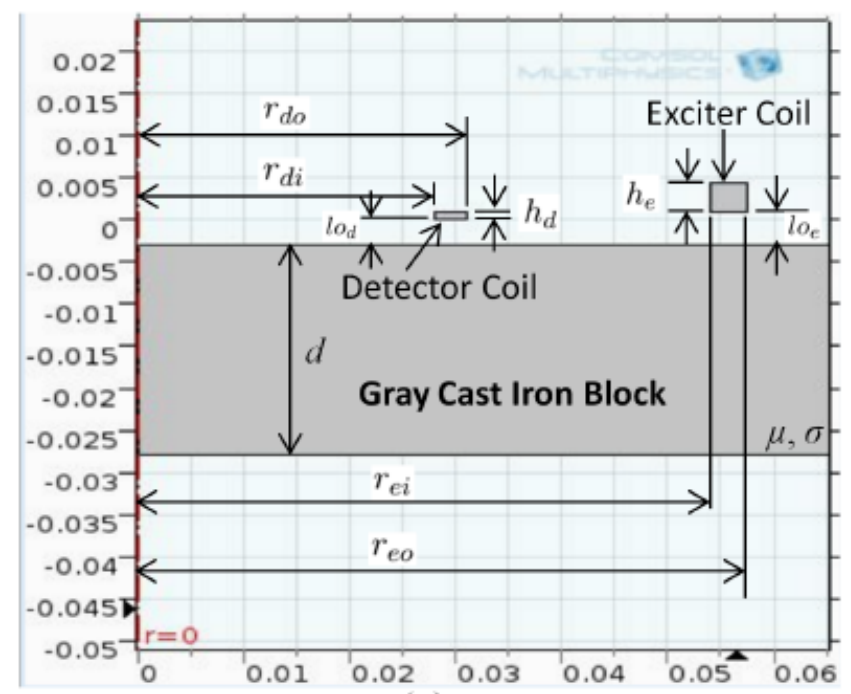

Fig. 5. 2D Axisymmetric model of the PEC sensor placed on a cast iron block.

using COMSOL Multiphysics ${ }^{\circledR}$. The parameters needed for simulation are given in Table I. Values of fixed parameters (or constants) we used for simulation are provided in Table II.

For simplicity we took the heights of exciter and detector coils $\left(h_{e}\right.$ and $\left.h_{d}\right)$ and vertical offsets of the two coils $\left(l_{e}\right.$ and $\left.l o_{d}\right)$ to be fixed. Since we use copper wires to wind coils, we could use standard permeability and conductivity of copper $\left(\mu_{e}, \mu_{d}, \sigma_{e}\right.$ and $\left.\sigma_{d}\right)$ for simulation. In addition, rough estimates of permeability $(\mu)$ and electrical conductivity $(\sigma)$ of the material being tested (cast iron in our case) are required. As given in Table II, the approximated values we used for cast iron were $\mu=4 \pi \times 10^{-5} \mathrm{H} / \mathrm{m}$ and $\sigma=2 \times 10^{6} \mathrm{~S} / \mathrm{m}$. If using precise values is desired, coupons of the material being tested can be extracted and those material properties can be experimentally measured using a Physical Property Measurement System (PPMS) [11] or by other suitable means. Alternatively, an approximation for those properties can be obtained from literature; reference [12] has published such information related to cast irons. We also took the amplitude of the excitation current pulse to be fixed at $200 \mathrm{~mA}$. As we designed the excitation circuit to produce a voltage pulse having $10 \mathrm{~V}$ amplitude in Subsection III-A, fixing the current amplitude at $200 \mathrm{~mA}$ meant the exciter coil resistance required to be $50 \Omega$, i.e., $R_{e}=50 \Omega$. Due to availability we chose the standard copper wire having $0.315 \mathrm{~mm}$ diameter to wind both exciter and detector coils. Therefore in our simulation $d_{e}=d_{d}=0.315 \mathrm{~mm}$. As we explain in Subsection III-C, we connect the detector coil output directly to an instrumentation amplifier having high input impedance. This results in the effective impedance $\left(Z_{d l}\right)$ felt by the detector coil be $\infty$. Now the objective was to estimate suitable inner and outer radii of both exciter and detector coils (i.e., $r_{e i}, r_{e o}, r_{d i}$ and $r_{d o}$ ) along with their respective number of coil turns (i.e., $n_{e}$ and $n_{d}$ ) in order to have cast iron thickness sensitivity from 5 to $25 \mathrm{~mm}$. Ideally this could have been formulated as an optimization problem and solved, but due to time constraints we conducted several trial and error experiments and decided upon the values provided in Table III to be used for sensor fabrication as those values yielded the necessary thickness sensitivity.

TABLE I

PARAMETERS REQUIRED FOR SIMULATION.

\begin{tabular}{c|c}
\hline Symbol & Description \\
\hline$r_{d i}$ & Inner radius of detector coil domain \\
\hline$r_{d o}$ & Outer radius of detector coil domain \\
\hline$h_{d}$ & Height of detector coil domain \\
\hline$l_{d}$ & Vertical offset of the detector coil \\
\hline$n_{d}$ & Number of detector coil turns \\
\hline$d_{d}$ & Diameter of the detector coil wire \\
\hline$\sigma_{d}$ & Electrical conductivity of the detector coil \\
\hline$\mu_{d}$ & Magnetic permeability of the detector coil \\
\hline$r_{e i}$ & Inner radius of exciter coil \\
\hline$r_{e o}$ & Outer radius of exciter coil \\
\hline$h_{e}$ & Height of exciter coil domain \\
\hline$l_{e}$ & Vertical offset of the exciter coil \\
\hline$n_{e}$ & Number of excitation coil turns \\
\hline$d_{e}$ & Diameter of the excitation coil wire \\
\hline$R_{e}$ & Resistance of the excitation coil wire \\
\hline$\sigma_{e}$ & Electrical conductivity of the exciter coil \\
\hline$\mu_{e}$ & Magnetic permeability of the exciter coil \\
\hline$d$ & Plate thickness \\
\hline$\sigma$ & Electrical conductivity of pipe material \\
\hline$\mu$ & Nonlinear magnetic permeability of pipe material \\
\hline$I_{e}$ & Amplitude or the excitation current pulse \\
\hline$Z_{d l}$ & Load impedance connected to the detector coil \\
\hline
\end{tabular}

TABLE II

FIXED PARAMETERS (CONSTANTS) USED FOR SIMULATION

\begin{tabular}{c|c}
\hline Symbol & Value \\
\hline$h_{e}=h_{d}$ & $10 \mathrm{~mm}$ \\
\hline$l o_{e}=l o_{d}$ & $2 \mathrm{~mm}$ \\
\hline$d_{e}=d_{d}$ & $0.315 \mathrm{~mm}$ \\
\hline$\sigma_{e}=\sigma_{d}$ & $5.998 \times 10^{7} \mathrm{~S} / \mathrm{m}$ as we use copper coils \\
\hline$\mu_{e}=\mu_{d}$ & $4 \pi \times 10^{-7} \mathrm{H} / \mathrm{m}$ as we use copper coils \\
\hline$\sigma$ & $2 \times 10^{6} \mathrm{~S} / \mathrm{m}$ \\
\hline$\mu$ & $4 \pi \times 10^{-5} \mathrm{H} / \mathrm{m}$ \\
\hline$I_{e}$ & $200 \mathrm{~mA}$ \\
\hline$R_{e}$ & $50 \Omega$ \\
\hline$Z_{d l}$ & $\infty$ \\
\hline
\end{tabular}


TABLE III

ESTIMATED PARAMETER VALUES USED TO FABRICATE THE PEC SENSOR.

\begin{tabular}{c|c}
\hline Symbol & Value \\
\hline$r_{d i}$ & $25 \mathrm{~mm}$ \\
\hline$r_{d o}$ & $28 \mathrm{~mm}$ \\
\hline$n_{d}$ & 300 \\
\hline$r_{e i}$ & $50 \mathrm{~mm}$ \\
\hline$r_{e o}$ & $57 \mathrm{~mm}$ \\
\hline$n_{e}$ & 600 \\
\hline \multicolumn{2}{|c}{}
\end{tabular}

We used $r_{d i}, r_{d o}, h_{d}, l o_{d}, r_{e i}, r_{e o}, h_{e}$ and $l o_{e}$ parameters to design a circular core using the SOLIDWORKS ${ }^{\circledR}$ software to hold the coils and 3D printed it using Polylactide (PLA) biodegradable polyester. We then wound the necessary numbers of turns $\left(n_{e}\right.$ and $\left.n_{d}\right)$ using copper wire having $0.315 \mathrm{~mm}$ wire diameter. Fig 6 shows the fabricated PEC sensor.

\section{Signal Acquisition}

The signal induced in the detector coil is in the order of millivolts, therefore we feed it through an instrumentation amplifier with a voltage gain of approximately 1000 before sampling the signal using the DAQ. We used the INA128P instrumentation amplifier Integrated Circuit (IC) for the purpose and Fig. 7 shows the schematic of the circuit we developed. $\mathrm{V}+$ and $\mathrm{V}$ - denote the $\pm 5 \mathrm{~V}$ DC supply provided to the IC. Ref is the reference from which the output voltage (i.e., the amplified PEC signal) is measured from, as done with the excitation amplifier (Fig. 4), we use the DAQ ground as the reference for reception as well.

We allowed the DAQ to sample signals at a rate of 100 $\mathrm{kHz}$. Since we capture a signal for a period of $50 \mathrm{~ms}$, a single signal results in 5000 samples. To reduce noise of detected signals, when taking a measurement we decided to excite the sensor with 10 consecutive pulses and take the average of the 10 detector coil signals resulting form the 10 falling edges of the pulses. Thus it takes about one second to

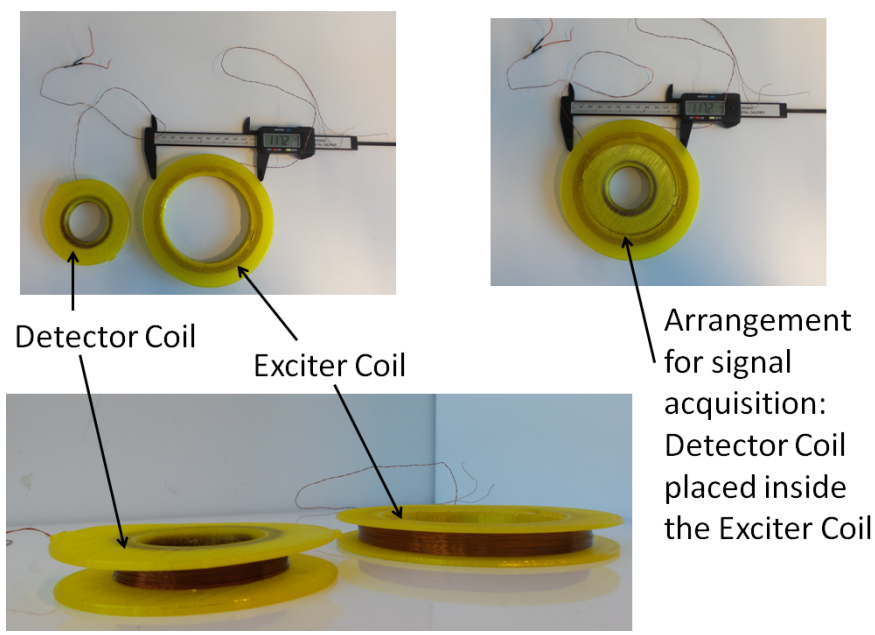

Fig. 6. Fabricated PEC sensor.

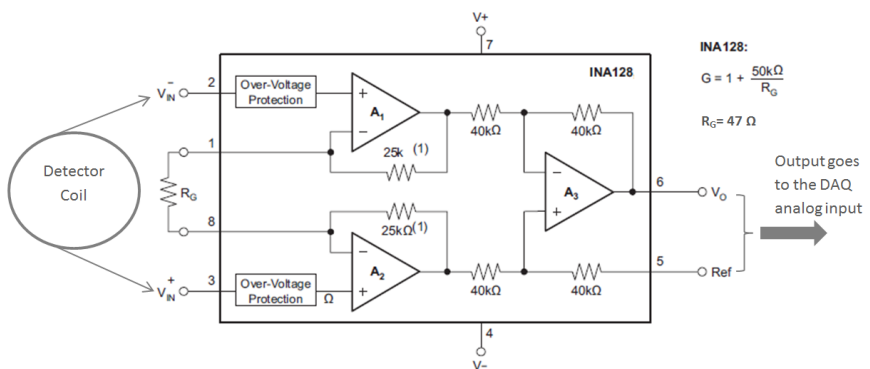

Fig. 7. Schematic of the instrumentation amplifier circuit for PEC signal reception, adapted from [13].

acquire a measurement. Averaging signals in this manner is commonly used in literature for PEC signal noise reduction [6]. Signals sampled by the DAQ are stored in the PC and the PC computes averaged responses from 10 signals acquired for one measurement. We use this averaged response to obtain the signal feature $\beta$ presented in Eq. 2 in order to study the functional behavior between cast iron thickness and the signal feature.

\section{PEC Signal Feature Extraction From EXPERIMENTAL SIGNALS}

Shown in Fig. 8 are averaged PEC signals in the form of $\ln [V(t)]$ plotted against time $t$ for different thicknesses of cast iron. We used cast iron blocks of thickness $7 \mathrm{~mm}$, $11 \mathrm{~mm}, 15 \mathrm{~mm}, 19 \mathrm{~mm}$ and $21 \mathrm{~mm}$ which were machined from an exhumed gray cast iron pipe. Length and width of a block was approximately $28 \mathrm{~mm}$ and $25 \mathrm{~mm}$ respectively. For comparison, an air signal is included in Fig 8. To examine repeatability and stability, we performed three measurements on each thickness and results from all three measurements are included in Fig. 8. As the thickness increases, we can see the discrimination between signals reducing as the penetration limit of the sensor is being reached and we see that the signals for thicknesses 19 and $21 \mathrm{~mm}$ are very close. However, the feature value $\beta$ for the two thicknesses are still discriminative as shown in Section V.

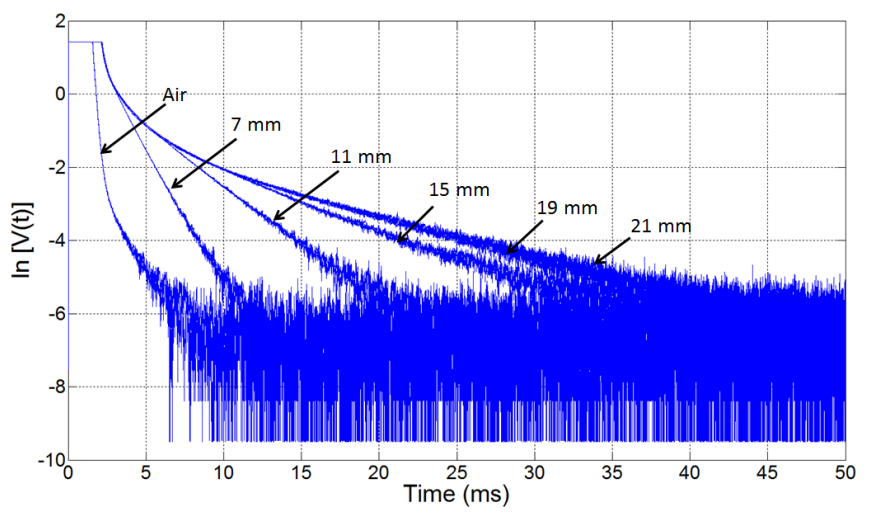

Fig. 8. PEC signals from the setup in the form of $\ln [V(t)]$ for different thicknesses of cast iron. 
Though the theory indicates that the $\beta$ feature has to be captured as time $t$ tends to $\infty$ in Eq. 2, as can be seen from Fig. 8, the signals enter the noise bound (i.e., $\ln [V(t)]<-5$ ) of the signal sampling circuitry as time increases. Therefore, we select the later stage of signals (i.e., $-4.5<\ln [V(t)]<$ $-2)$ to extract $\beta$. In other words, we consider that region of any signal produced by our setup to indicatively be the $t \approx \infty$ region for practical purposes.

By looking at Eq. 2 it can be understood that the feature $\beta$ is simply the reciprocal of the absolute of the gradient of $\ln [V(t)]$ when $t \rightarrow \infty$. Therefore, to extract $\beta$ we estimate the gradient of the linear region encapsulated within $-4.5<\ln [V(t)]<-2$ of our experimental signals. This is done by running the Random Sample Consensus (RANSAC) algorithm [14] on the $-4.5<\ln [V(t)]<-2$ signal region as it identifies the most linear portion of a given dataset and is minimally influenced by outliers. Fig. 9 shows the RANSAC fitted straight lines to all the experimental signals shown in Fig. 8. Iteration Number $=1000$, Distance Threshold $=0.001$ and Distance Threshold Ratio $=0.01$ were the parameters used when running the RANSAC algorithm.

\section{EXPERIMENTAL RESULTS}

By plotting the variation of cast iron thickness $d$ taken in $\mathrm{m}$ against $\beta$, it can be seen that the thickness behaves as a function of the feature. Fig. 10 and Fig. 11 are two examples on how the function may be characterized. Both figures display feature values form three measurements performed on each thickness in order to depict repeatability and stability. The variation of $\ln (d)$ against $\ln (\beta)$ shown in Fig. 10 is fairly linear indicating that it could be analytically modeled as a straight line. A nonlinearity can been seen at the high thickness end due to the sensor's penetration capability being reached. The function shown in Fig. 11, i.e., variation of $d^{2}$ against $\beta$ is nonlinear. Such a function may be modeled as a nonlinear regression function using techniques such as Gaussian Process (GP) regression as done in [9], [15], [16], [17]. Despite how a function may be modeled, the prevalence of a functional

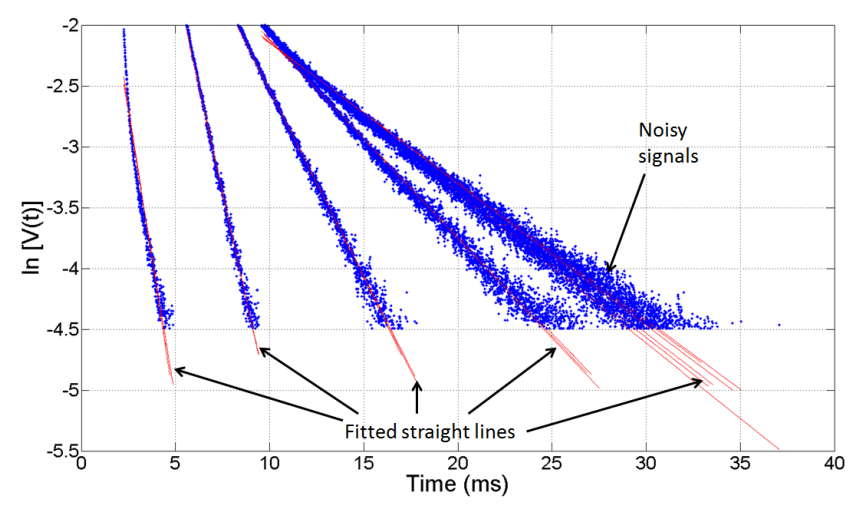

Fig. 9. RANSAC fitted lines to estimate the feature value $\beta$ from the PEC signal region $-4.5<\ln [V(t)]<-2$. behavior between cast iron thickness and the $\beta$ feature is the important and desirable aspect. This indicates that the function can be calibrated for a particular material using several calibration thicknesses and hence the proposed PEC sensing setup can be used for cast iron NDE applications.

The $\beta$ feature was also observed to be lowly influenced by lift-off. We repeated the experiment on the same cast iron blocks by placing the sensor on a $12 \mathrm{~mm}$ thick layer of perspex resting on the blocks. This effectively created a $12 \mathrm{~mm}$ liftoff between the sensor and a calibration block. The $\beta$ values obtained with liftoff were plotted against thickness as shown

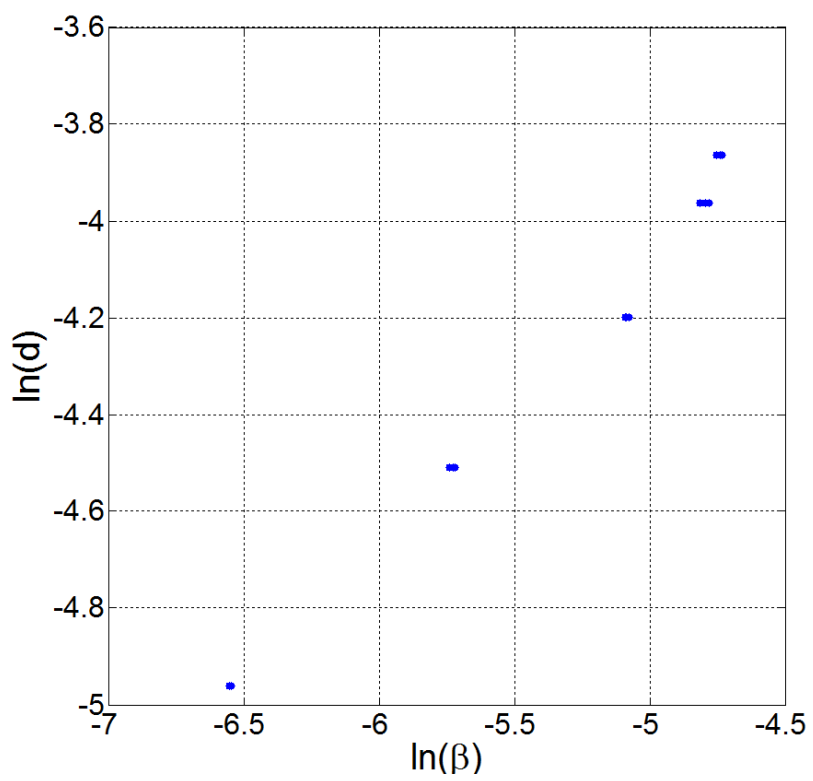

Fig. 10. Cast iron thickness $d$ in $\mathrm{m}$ behaving as a function of the feature $\beta$ when plotted as $\ln (d)$ against $\ln (\beta)$.

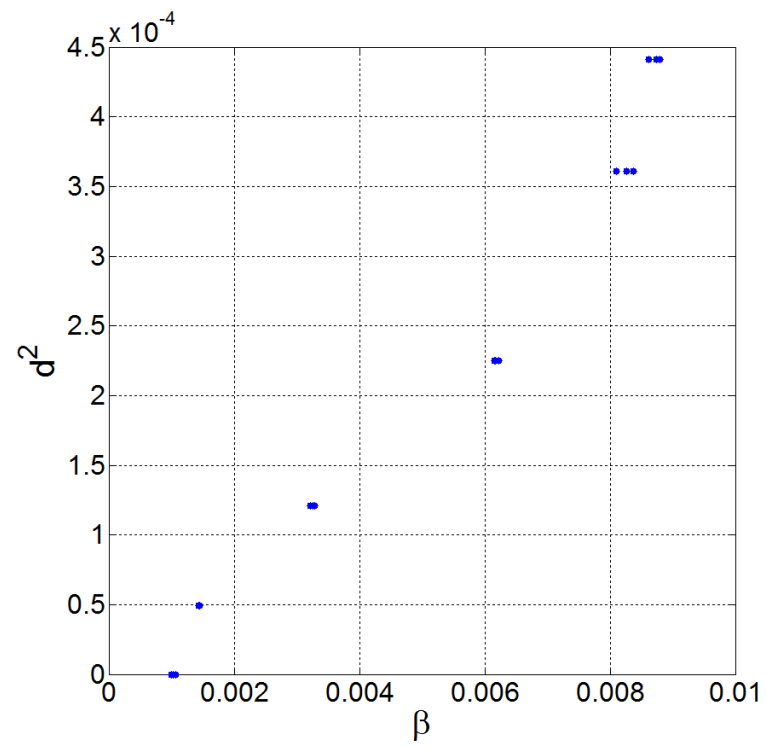

Fig. 11. Cast iron thickness $d$ in $\mathrm{m}$ behaving as a function of the feature $\beta$ when plotted as $d^{2}$ against $\beta$ ( $d^{2}=0$ corresponds to the air signal). 
in Fig. 12. A low average variation of less than $2 \%$ in $\ln (\beta)$ was observed with respect to the zero lift-off case. This is again desirable as being lowly influenced by lift-off suggest that the $\beta$ feature is usable on applications such as in-situ pipe assessment where pipe surfaces may be covered by a protective layer, dust, rust or graphitization.

\section{CONCLUSION}

Although literature has investigated using PEC sensor for thickness assessment of structural steels, work investing sensor performance on cast irons are rare. Therefore in this paper we presented steps for designing a PEC sensing setup which can measure cast iron thickness up to about $25 \mathrm{~mm}$ and evaluated its performance. Steps for designing the sensor excitation circuit, PEC sensor, and the signal acquisition circuit were presented. We also presented a unique PEC signal processing method which yields a signal feature which exhibits function behavior with cast iron thickness. The thickness-feature function can be characterized and modeled in different ways and the prevalence of the functional behavior suggests that PEC sensors can be used for cast iron thickness assessment provided that the thickness feature function can be calibrated. The signal feature has a desirable characteristic of being lowly influenced by lift-off indicating that the developed setup has usability for NDE applications such as in-situ cast iron water pipe assessment.

\section{ACKNOWLEDGMENT}

This work is an outcome from the Critical Pipes Project funded by Sydney Water Corporation and University of Technology Sydney.

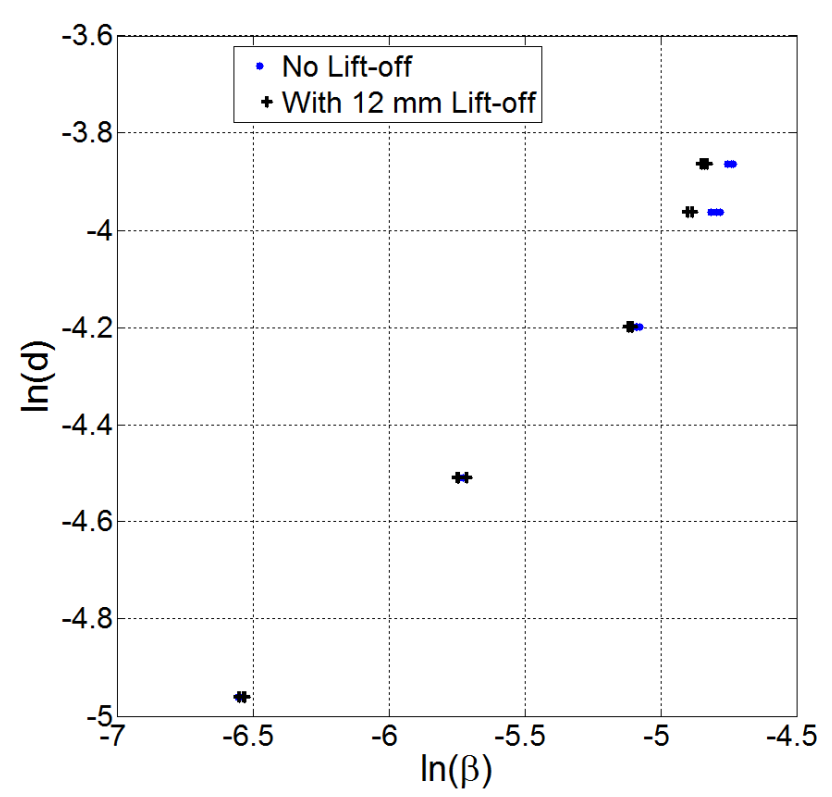

Fig. 12. Low influence from lift-off on $\beta$.

\section{REFERENCES}

[1] J. García-Martín, J. Gómez-Gil, and E. Vázquez-Sánchez, "Nondestructive techniques based on eddy current testing," Sensors, vol. 11, no. 3, pp. 2525-2565, 2011.

[2] A. M. N. N. B. Ulapane, "Nondestructive evaluation of ferromagnetic critical water pipes using pulsed eddy current testing," $\mathrm{Ph}$.D. dissertation, 2016.

[3] C. Huang, W. Xinjun, X. Zhiyuan, and Y. Kang, "Pulsed eddy current signal processing method for signal denoising in ferromagnetic plate testing," NDT \& E International, vol. 43, no. 7, pp. 648-653, 2010.

[4] C. Huang, X. Wu, Z. Xu, and Y. Kang, "Ferromagnetic material pulsed eddy current testing signal modeling by equivalent multiple-coilcoupling approach," NDT \& E International, vol. 44, no. 2, pp. 163-168, 2011.

[5] X. Chen and Y. Lei, "Excitation current waveform for eddy current testing on the thickness of ferromagnetic plates," NDT \& E International, vol. 66, pp. 28-33, 2014.

[6] C. Huang and X. Wu, "An improved ferromagnetic material pulsed eddy current testing signal processing method based on numerical cumulative integration," NDT \& E International, vol. 69, pp. 35-39, 2015.

[7] J. V. Miro, J. Rajalingam, T. Vidal-Calleja, F. de Bruijn, R. Wood, D. Vitanage, N. Ulapane, B. Wijerathna, and D. Su, "A live test-bed for the advancement of condition assessment and failure prediction research on critical pipes," in Proceedings of the Leading-Edge Strategic Asset Management Conference (LESAM13), 2013.

[8] R. Petersen and R. Melchers, "Long-term corrosion of cast iron cement lined pipes," Corrosion and Prevention, vol. 23, p. 10, 2012.

[9] N. Ulapane, A. Alempijevic, T. Vidal-Calleja, J. V. Miro, J. Rudd, and M. Roubal, "Gaussian process for interpreting pulsed eddy current signals for ferromagnetic pipe profiling," in 2014 9th IEEE Conference on Industrial Electronics and Applications. IEEE, 2014, pp. 1762-1767.

[10] A. Sophian, "Characterisation of surface and sub-surface discontinuities in metals using pulsed eddy current sensors," Ph.D. dissertation, University of Huddersfield, 2003.

[11] J. Lashley, M. Hundley, A. Migliori, J. Sarrao, P. Pagliuso, T. Darling, M. Jaime, J. Cooley, W. Hults, L. Morales et al., "Critical examination of heat capacity measurements made on a quantum design physical property measurement system," Cryogenics, vol. 43 , no. 6 , pp. $369-378$, 2003.

[12] J. R. Davis et al., ASM specialty handbook: cast irons. ASM international, 1996.

[13] T. Instruments, "Texas instruments incorporated ina128 datasheet," 2009.

[14] M. A. Fischler and R. C. Bolles, "Random sample consensus: a paradigm for model fitting with applications to image analysis and automated cartography," Communications of the ACM, vol. 24, no. 6, pp. 381-395, 1981.

[15] N. N. Ulapane and S. G. Abeyratne, "Gaussian process for learning solar panel maximum power point characteristics as functions of environmental conditions," in 2014 9th IEEE Conference on Industrial Electronics and Applications. IEEE, 2014, pp. 1756-1761.

[16] K. Thiyagarajan, S. Kodagoda, and N. Ulapane, "Data-driven machine learning approach for predicting volumetric moisture content of concrete using resistance sensor measurements," in Industrial Electronics and Applications (ICIEA), 2016 IEEE 11th Conference on. IEEE, 2016, pp. 1288-1293.

[17] C. E. Rasmussen, "Gaussian processes for machine learning," 2006. 